УДК 544.6.018.47-039.6+546.865-31

DOI: https://doi.org/10.17308/kcmf.2019.21/758

Поступила в редакцию 30.04.2019

Подписана в печать 15.05.2019

\title{
ДИЭЛЕКТРИЧЕСКАЯ РЕЛАКСАЦИЯ И ПРОТОННАЯ ПРОВОДИМОСТЬ ПОЛИСУРЬМЯНОЙ КИСЛОТЫ, ДОПИРОВАННОЙ ИОНАМИ ВАНАДИЯ
}

\author{
(c) 2019 Л. Ю. Коваленко $\bowtie$, В. А. Бурмистров \\ Челябинский государственный университет \\ ул. Бр. Кашириных, 129, 454001 Челябинск, Российская Федерация
}

\begin{abstract}
Аннотация. Методом импедансной спектроскопии исследованы протонпроводящие свойства полисурьмяной кислоты (ПСК), допированной ионами ванадия. Для твердых растворов состава $\mathrm{H}_{2} \mathrm{Sb}_{2-x} \mathrm{~V}_{x} \mathrm{O}_{6} \cdot \mathrm{nH}_{2} \mathrm{O}$, кристаллизующихся в структурном типе пирохлора (пр. гр. симм. $F d 3 m$ ), показано, что увеличение количества ванадия в образце приводит к росту удельной протонной проводимости, которая для крайнего твердого раствора замещения $\mathrm{H}_{2} \mathrm{Sb}_{1.52} \mathrm{~V}_{0.48} \mathrm{O}_{6} \cdot \mathrm{nH}_{2} \mathrm{O}$ составляет $66 \mathrm{mCм} / \mathrm{M}$. Из анализа данных диэлектрической спектроскопии при температурах 218-298 К определена энергия активации проводимости, которая составила 30ะ2 КДж/моль. Предложен механизм протонного транспорта, согласно которому в допированных ионами ванадия ПСК проводимость осуществляется по системе водородных связей, образованных молекулами воды, расположенными в гексагональных каналах структуры типа пирохлора, и анионами кислорода октаэдра, формирующего каркас структуры.
\end{abstract}

Ключевые слова: твёрдые электролиты, структура типа пирохлора, соединения сурьмы, полисурьмяная кислота, твёрдые растворы замещения, протонная проводимость.

\section{ВВЕДЕНИЕ}

Интерес многих исследователей направлен на синтез неорганических веществ, обладающих высокими значениями протонной проводимости при низких температурах [1, 2]. Введение таких компонентов в состав протонпроводящих перфторированных сульфосодержащих мембран приводит к повышению значений проводимости на несколько порядков [3-5].

Одним из перспективных соединений является полисурьмяная кислота (ПСК) состава $\mathrm{H}_{2} \mathrm{Sb}_{2} \mathrm{O}_{6} \cdot \mathrm{nH}_{2} \mathrm{O}(2<\mathrm{n}<4)$, кристаллизующаяся в структурном типе пирохлора (пр. гр. симм. $F d 3 m$ ). При распределении атомов ПСК по кристаллографическим позициям структуры типа пирохлора вакантными остаются 8bпозиции, в результате чего формируется ажурный каркас дефектной структуры, состоящий из $\left[\mathrm{SbO}_{3}\right]^{-}$- октаэдров, соединенных вершинами, и имеющий каналы с гексагональными полостями, в которых расположены протоны [6].

В литературе большое внимание уделено описанию механизмов транспорта протонов в ПСК при различных температурах [7, 8]. Пока-

\footnotetext{
$\triangle$ Коваленко Лилия Юрьевна,

e-mail: LKovalenko90@mail.ru
}

зано, что протонная проводимость $\mathrm{H}_{2} \mathrm{Sb}_{2} \mathrm{O}_{6} \cdot \mathrm{nH}_{2} \mathrm{O}$ обусловлена структурными особенностями, наличием гексагональных каналов, по которым осуществляется перескок протонов с молекул воды на анионы кислорода каркаса $\left[\mathrm{SbO}_{3}\right]^{-}$. При этом молекулы воды и анионы кислорода, расположенные в позициях 8b и $48 \mathrm{f}$ соответственно, образуют непрерывную зигзагообразную цепочку в кристаллографическом направлении типа $<111>$ [7].

Улучшить транспортные свойства ПСК можно, уменьшив энергию взаимодействия протонов с анионным остовом и создавая пути их миграции по системе водородных связей, образуемых молекулами воды. Этого можно достичь путем частичного замещения ионов сурьмы на менее электроотрицательные атомы [1,9].

В связи с этим целью настоящей работы являлся синтез и исследование транспорта протонов в ПСК, допированной ионами ванадия.

\section{ЭКСПЕРИМЕНТАЛЬНАЯ ЧАСТЬ}

Синтез образцов проводили методом соосаждения, добавляя по каплям раствор ванадата натрия заданной концентрации к раствору трёххлористой сурьмы, предварительно окислен- 
ной азотной кислотой, по методике, описанной в [10]. Полученный осадок отделяли от маточного раствора, промывали дистиллированной водой до отрицательной реакции фильтрата на ионы хлора, высушивали на воздухе и выдерживали длительное время при комнатной температуре в обычных условиях ( $T=298 \mathrm{~K}, \mathrm{RH} \sim 60 \%)$. После этого образец перетирали в агатовой ступке, просеивали через сита с ячейками 0.1 и $0.2 \mathrm{~mm}$, средний размер частиц составил $0.15 \mathrm{~mm}$.

Соотношения ванадия и сурьмы (at. \%) в образцах находили с учетом данных рентгенофлуоресцентного спектрометра ARL QuanT'X, чувствительность прибора <1 ppm.

Структурные исследования фаз выполнили на рентгеновском дифрактометре Rigaki ultima IV (фильтрованное CuK $\alpha$-излучение) в диапазоне углов дифракции $10^{\circ}<2 \theta<80^{\circ}$. Анализировали законы погасания максимумов, изменение их интенсивности и межплоскостные расстояния. По данным рентгеновских исследований методом Ритвельда с использованием программы PowderCell 2.4 было определено расположение ионов по правильной системе точек структуры типа пирохлора пр. гр. симм. Fd3m, а также параметр элементарной ячейки [10].

Размер областей когерентного рассеяния (ОКР), определенный по формуле Дебая-Шеррера по рефлексу 8.0.0., составил $40 \mathrm{~nm}$.

В предыдущих работах $[10,11]$ было показано, что допирование ПСК ионами ванадия в рамках структуры типа пирохлора имеет концентрационное ограничение: твёрдый раствор замещения $\mathrm{H}_{2} \mathrm{Sb}_{2-x} \mathrm{~V}_{x} \mathrm{O}_{6} \cdot \mathrm{nH}_{2} \mathrm{O}$ формируется при $0<\mathrm{x}<0.48$. Поэтому в качестве объектов исследования были выбраны мелкодисперсные порошки, содержащие по данным элементного анализа $0 ; 10(\mathrm{x}=0.2) ; 20(\mathrm{x}=0.40) ; 24(\mathrm{x}=0.48)$ at. \% ванадия.

Протонпроводящие свойства исследовали с помощью импедансметра Elins-Z1000J в диапазоне частот $1 \mathrm{~Hz}-2 \mathrm{MHz}$. Для этого образцы спрессовывали при одинаковом давлении в виде таблетки в специально изготовленной ячейке (использовали графитовые электроды, диаметр каждого электрода $-2.820 \pm 0.005 \mathrm{~mm}$ ). Площадь образца оценивали по площади поверхности электрода. Измерительное напряжение составляло $100 \mathrm{mV}$, считали, что приложенное поле не вызывает заметных нелинейных искажений поляризации [12]. Относительную влажность 58 \% задавали путем выдерживания ячейки в колбе над зеркалом насыщенного раствора соли $\mathrm{NaBr}$. Для исследования проводимости при понижен- ных температурах ячейку предварительно выдерживали в колбе над зеркалом насыщенного раствора соли $\mathrm{NaBr}$ в течение суток, после чего помещали в термостат, позволяющий при помощи охлаждения твердой углекислотой изменять температуру от 218 до 298 К. Точность измерения температуры составила $\pm 1 \mathrm{~K}$.

По данным измерения действительной $Z^{\prime}$ и мнимой $Z$ " части импеданса определили диэлектрические характеристики по формулам:

$$
\begin{gathered}
\varepsilon^{\prime}=\frac{1}{2 \pi f C_{0}} \cdot \frac{Z^{\prime \prime}}{\left(Z^{\prime}\right)^{2}+\left(Z^{\prime \prime}\right)^{2}}, \\
\varepsilon^{\prime \prime}=\frac{1}{2 \pi f C_{0}} \cdot \frac{Z^{\prime}}{\left(Z^{\prime}\right)^{2}+\left(Z^{\prime \prime}\right)^{2}}, \\
\operatorname{tg} \delta=\frac{\varepsilon^{\prime \prime}}{\varepsilon^{\prime}}, \\
M^{\prime \prime}=2 \pi f C_{0} Z^{\prime},
\end{gathered}
$$

и вычислили величину удельной проводимости:

$$
\sigma=\frac{d}{S} \cdot \frac{Z^{\prime}}{\left(Z^{\prime}\right)^{2}+\left(Z^{\prime \prime}\right)^{2}}
$$

где $f$ - частота электрического поля; $\varepsilon^{\prime}$ и $\varepsilon^{\prime \prime}$ действительная и мнимая часть диэлектрической проницаемости $\varepsilon^{*} ; M^{\prime \prime}$ - мнимая часть электрического модуля $M^{*} ; \sigma$ - удельная проводимость; $\operatorname{tg} \delta$ - тангенс угла диэлектрических потерь; $C_{0}$ - геометрическая емкость:

$$
C_{0}=\frac{\varepsilon_{0} S}{d},
$$

$\varepsilon_{0}=8.85 \cdot 10^{-12} \mathrm{~F} / \mathrm{m}$ (электрическая постоянная), $S=(6.243 \pm 0.007) \cdot 10^{-6} \mathrm{~m}^{2}$ (площадь поверхности электрода), $d=(2.000-2.500) \cdot 10^{-3} \mathrm{~m}$ (толщина таблетки).

\section{РЕЗУЛЬТАТЫ И ИХ ОБСУЖДЕНИЕ}

По данным РФА на рентгенограммах образцов присутствует одинаковый набор дифракционных максимумов, расположение и интенсивность которых соответствует фазе типа пирохлора [13]. Однако с увеличением количества ванадия наблюдается их сдвиг в область больших углов дифракции. Параметр элементарной ячейки уменьшается от 10.360 (ПСК) до $10.314 \AA$ $(x=0.48)[10]$.

Для описания протонпроводящих свойств допированных соединений использовали модель «зерен и прослоек» $[14,15]$. Считали, что дисперсный образец состоит из частиц, разделенных прослойками.

Годограф импеданса измерительной ячейки для температуры 298 К и RH = 58 \% представляет собой слегка вытянутую полуокруж- 
ность и прямую линию под небольшим углом к оси абсцисс (рис. 1). Это свидетельствует о наличии процессов релаксации заряда, протекающего вблизи электродов (низкочастотная область), преимущественно в прослойках (низкои среднечастотная область), и в самих частицах (высокочастотная область). Увеличение количества ионов ванадия приводит к сдвигу полуокружностей и к уменьшению их радиусов, что свидетельствует об увеличении проводимости образцов (рис. 1).

Для оценки влияния допирования на проводимость частиц на крайнем твердом растворе замещения $\mathrm{H}_{2} \mathrm{Sb}_{1.52} \mathrm{~V}_{0.48} \mathrm{O}_{6} \cdot \mathrm{nH}_{2} \mathrm{O}$ изучили изменение диэлектрических параметров при различных частотах и температурах. Исследования проводили при низких температурах, что позволило уменьшить вклад сквозной проводимости поверхности частиц в общую проводимость.

На зависимости $\varepsilon^{\prime}$ от частоты (рис. 2) можно выделить две области: низкочастотную, характеризующуюся большими значениями $\varepsilon^{\prime}\left(>10^{2}\right)$, и высокочастотную, характеризующуюся уменьшением значений $\varepsilon^{\prime}$ до некоторой постоянной величины. Такой вид зависимости можно объяснить смещением зарядов внутри частиц под воздействием поля и образованием макродиполей. При низких частотах релаксационная поляризация приводит к большому значению диэлектрической проницаемости, однако с ростом частоты подвижные ионы перестают успевать за изменениями направления поля и наблюдается уменьшение значений $\varepsilon^{\prime}$. Увеличение температуры приводит к возрастанию значений $\varepsilon^{\prime}$ в низкочастотной области (рис. 2) в связи с увеличением концентрации подвижных протонов. Такие изменения $\varepsilon^{\prime}$ характерны для дисперсных ионных проводников [16].

При увеличении температуры протоны в допированных образцах более подвижны, что подтверждается данными частотных зависимостей мнимой части $\varepsilon^{\prime \prime}$ диэлектрической проницаемости (рис. 3).

При низких частотах наблюдается монотонное уменьшение значений $\varepsilon^{\prime \prime}$, после чего на зависимостях $\varepsilon^{\prime \prime}$ от частоты фиксируются максимумы, что можно объяснить реализацией при этих частотах релаксационных процессов, связанных с транспортом протонов в частице. С увеличением температуры максимум сдвигается в высокочастотную область, это свидетельствует о большей подвижности протонов.

Более отчетливо релаксационные максимумы видны на зависимостях тангенса угла диэлектрических потерь $\operatorname{tg} \delta$ от частоты (рис. 4). При увеличении температуры наблюдается такое же смещение максимумов в область больших частот. Однако максимумы имеют большую ширину, что может быть связано с наличием нескольких времен релаксации протонов. Для под-

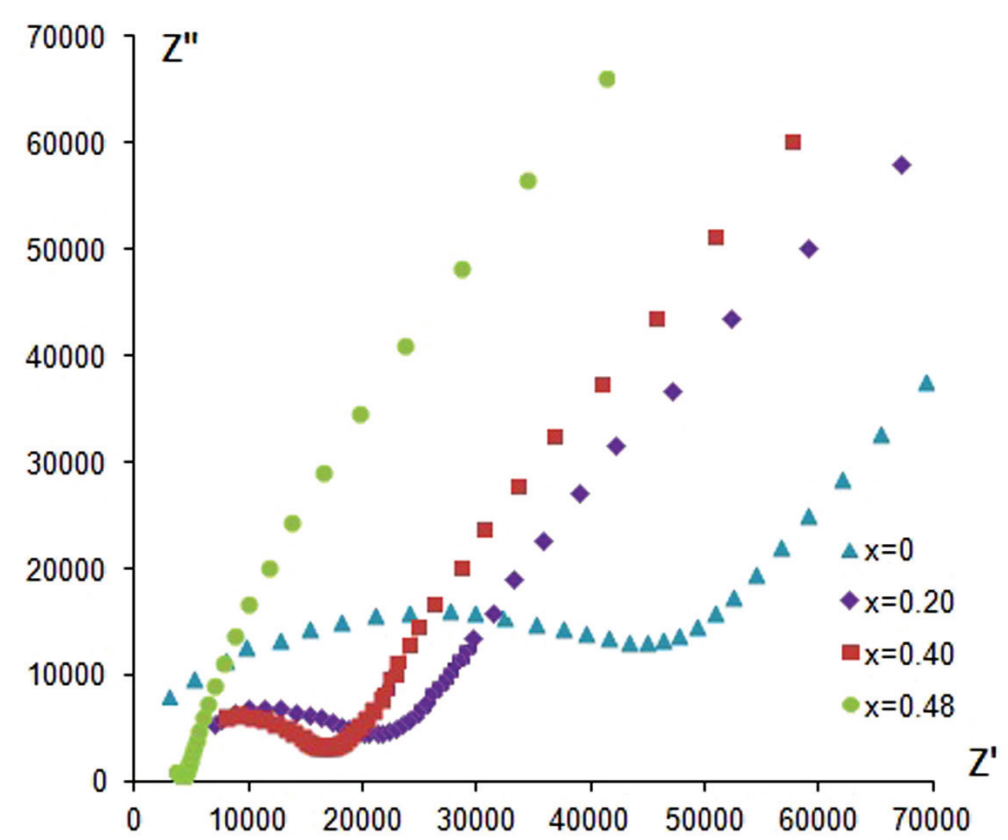

Рис. 1. Годограф импеданса $\mathrm{H}_{2} \mathrm{Sb}_{2-x} \mathrm{~V}_{x} \mathrm{O}_{6} \cdot \mathrm{nH}_{2} \mathrm{O}$, в которых $x$ равно $0 ; 0.20 ; 0.40 ; 0.48$

[Fig. 1. Impedance hodograph of $\mathrm{H}_{2} \mathrm{Sb}_{2-x} \mathrm{~V}_{x} \mathrm{O}_{6} \cdot \mathrm{nH}_{2} \mathrm{O}$, in which $x$ is $0 ; 0.20 ; 0.40 ; 0.48$ ] 


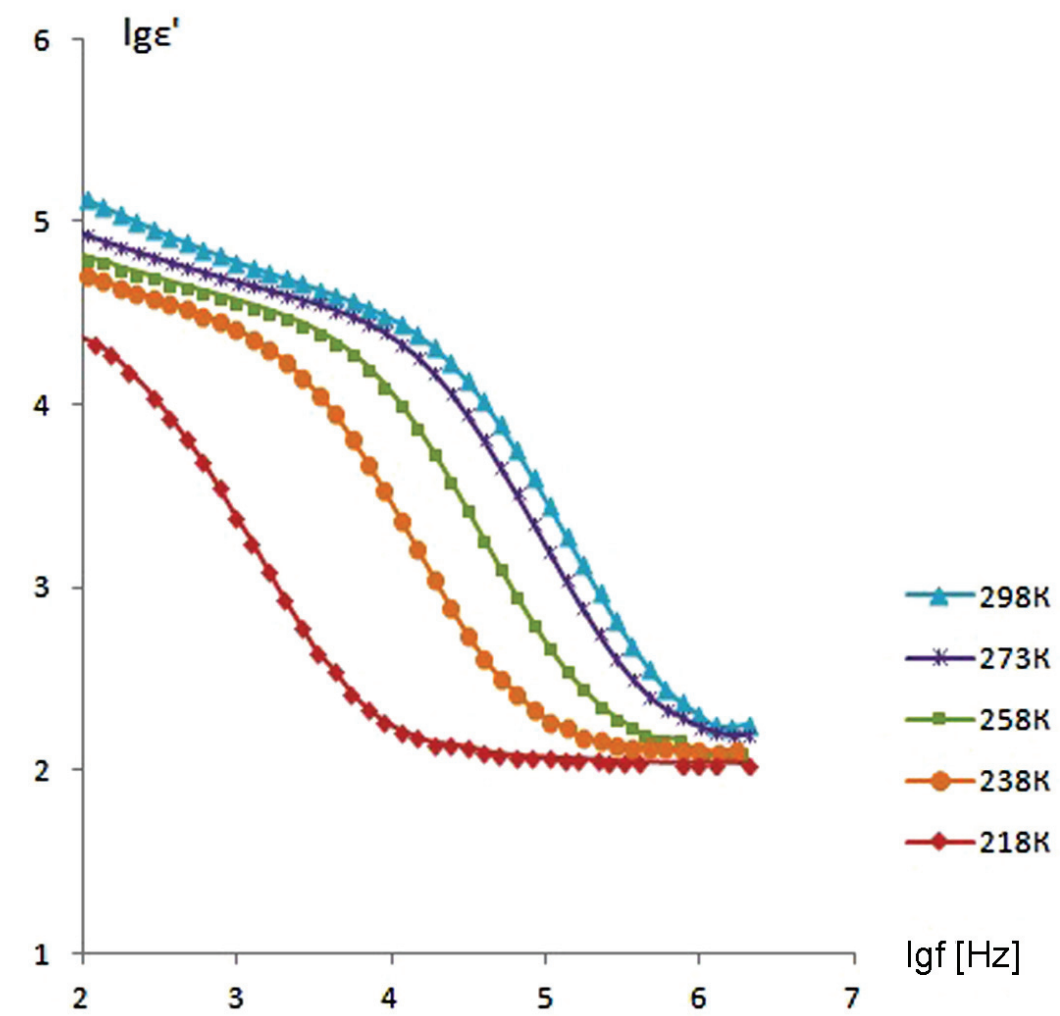

Рис. 2. Зависимость действительной части диэлектрической проницаемости от логарифма частоты при различных температурах для $\mathrm{H}_{2} \mathrm{Sb}_{1.52} \mathrm{~V}_{0.48} \mathrm{O}_{6} \cdot \mathrm{nH}_{2} \mathrm{O}$

[Fig. 2. Dependence of the real component of dielectric permeability on logarithm of frequency at different temperatures for $\left.\mathrm{H}_{2} \mathrm{Sb}_{1.52} \mathrm{~V}_{0.48} \mathrm{O}_{6} \cdot \mathrm{nH}_{2} \mathrm{O}\right]$

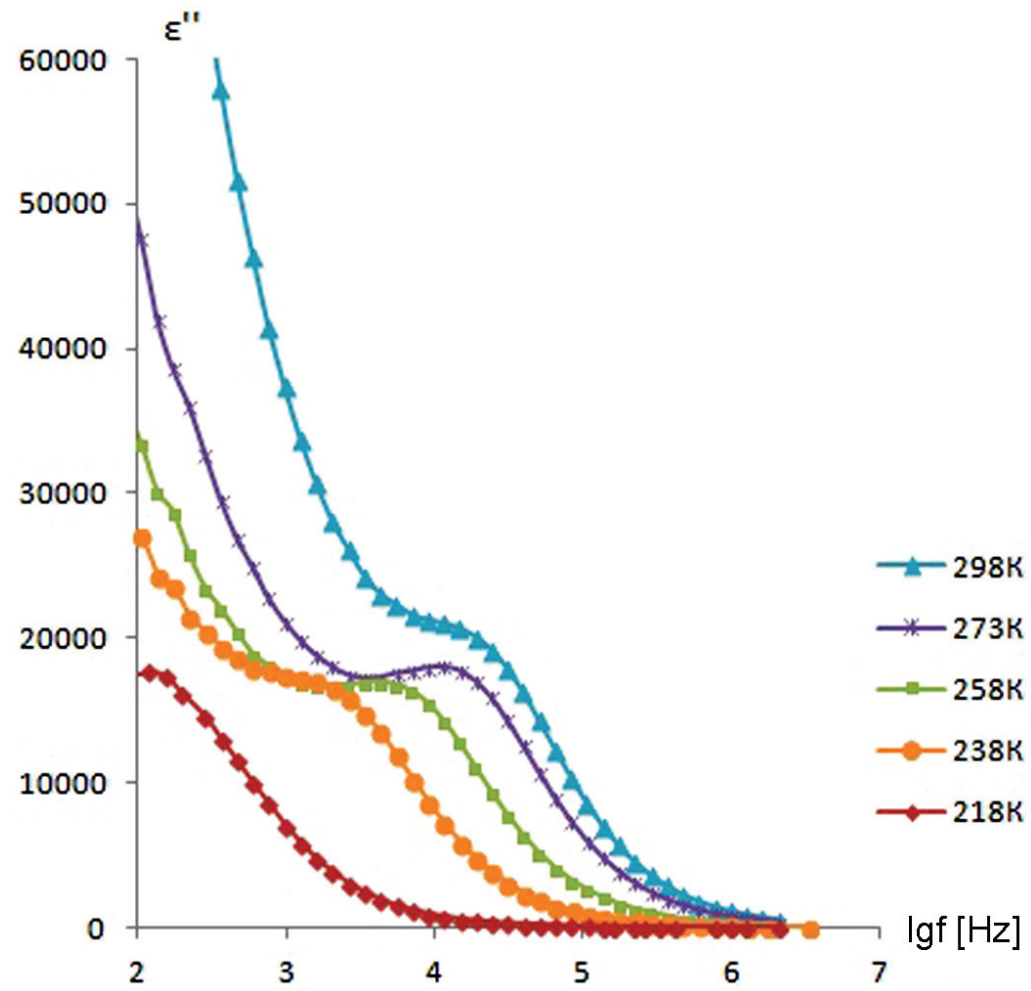

Рис. 3. Зависимость мнимой части диэлектрической проницаемости от логарифма частоты при различных температурах для $\mathrm{H}_{2} \mathrm{Sb}_{1.52} \mathrm{~V}_{0.48} \mathrm{O}_{6} \cdot \mathrm{nH}_{2} \mathrm{O}$

[Fig. 3. Dependence of the imaginary component of dielectric permeability on logarithm of frequency at different temperatures for $\left.\mathrm{H}_{2} \mathrm{Sb}_{1.52} \mathrm{~V}_{0.48} \mathrm{O}_{6} \cdot \mathrm{nH}_{2} \mathrm{O}\right]$ 


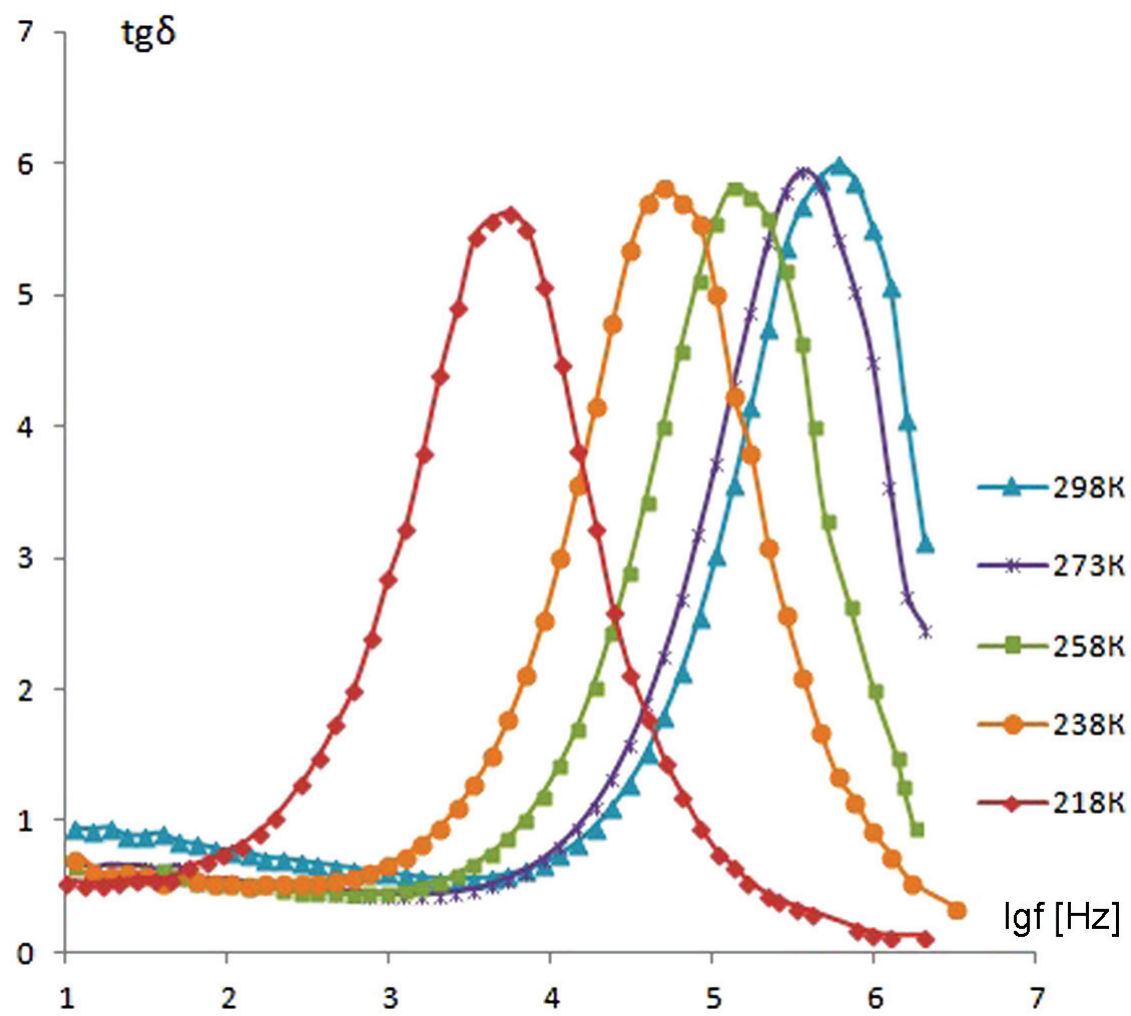

Рис. 4. Зависимость тангенса угла диэлектрических потерь от логарифма частоты при различных температурах для $\mathrm{H}_{2} \mathrm{Sb}_{1.52} \mathrm{~V}_{0.48} \mathrm{O}_{6} \cdot \mathrm{nH}_{2} \mathrm{O}$

[Fig. 4. Dependence of dielectric loss tangent on logarithm of frequency at different temperatures for

$$
\left.\mathrm{H}_{2} \mathrm{Sb}_{1.52} \mathrm{~V}_{0.48} \mathrm{O}_{6} \cdot \mathrm{nH}_{2} \mathrm{O}\right]
$$

тверждения данного предположения были проведены исследования электрического модуля от частоты [17].

На зависимостях $M^{\prime \prime}$ от частоты фиксируются максимумы (рис. 5), которые с увеличением температуры смещаются в область больших частот. С учетом координат максимумов зависимости были перестроены в $M^{\prime \prime} / M_{\max }^{\prime \prime}$ от $\lg \left(f / f_{\max }\right)$ (рис. 6) и найдены значения их полуширины $\delta\left(\lg \left(f / f_{\max }\right)\right)$.

Полученные кривые имеют одинаковую форму при различных температурах с близкой полушириной, равной $\delta\left(\lg \left(f / f_{\max }\right)\right)=1.6 \pm 0.2$ (рис. 6) и отличной от величины дебаевского максимума $\delta\left(\lg \left(f / f_{\max }\right)\right)=1.14$ [18]. Уширение свидетельствует о наличии нескольких времен релаксации протонов, что обусловлено различными размерами частиц.

На зависимости $\lg \sigma$ от частоты при различных температурах можно выделить два участка (рис. 7): низко- и среднечастотный, на котором наблюдается увеличение проводимости с ростом частоты, и высокочастотный, характеризующийся частотно независимым плато. Увеличение проводимости при небольших частотах может быть связано с образованием в приэлектро- дной области и на границах частиц объемного заряда, а постоянное значение проводимости при частотах от $10^{3}$ до $10^{6} \mathrm{~Hz}$ соответствует протонной проводимости частиц. По высокочастотному участку на зависимости $\lg \sigma$ от частоты были найдены значения протонной проводимости частиц (рис. 7) при различных температурах.

Логарифмическая зависимость проводимости от обратной температуры (рис. 8) представляет собой прямую линию с меньшим углом наклона по сравнению с ПСК, что характерно для допированных оксидных соединений [19]. Значение энергии активации составляет $30 \pm 2 \mathrm{KJ} / \mathrm{mol}$, что меньше энергии активации проводимости протонов ПСК, которая согласно [7], равна $50 \mathrm{KJ} / \mathrm{mol}$.

Повышение температуры от 218 до $298 \mathrm{~K}$ приводит к экспоненциальному возрастанию проводимости частиц и смещению максимумов на кривой $\operatorname{tg} \delta$ от частоты (рис. 4 ) в область больших частот, что обусловлено улучшением транспортных свойств протонов внутри частиц. Значение энергии активации, определенное по зависимости логарифма частоты, при котором фиксируется максимум на кривой $\operatorname{tg} \delta$ (рис. $8 b$ ), от обратной температуры, совпадает с энергией активации проводимости. 


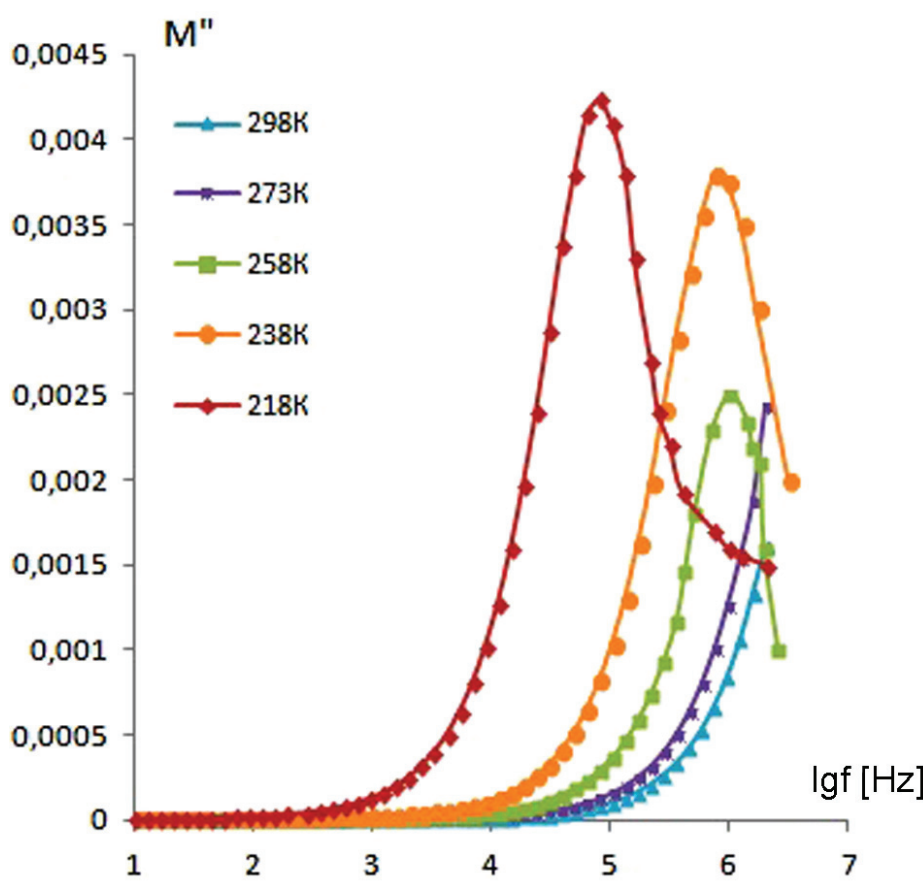

Рис. 5. Зависимость мнимой части электрического модуля от логарифма частоты при различных температурах для $\mathrm{H}_{2} \mathrm{Sb}_{1.52} \mathrm{~V}_{0.48} \mathrm{O}_{6} \cdot \mathrm{nH}_{2} \mathrm{O}$

[Fig. 5. Dependence of the imaginary component of electric module on logarithm of frequency at different temperatures for $\left.\mathrm{H}_{2} \mathrm{Sb}_{1.52} \mathrm{~V}_{0.48} \mathrm{O}_{6} \cdot \mathrm{nH}_{2} \mathrm{O}\right]$

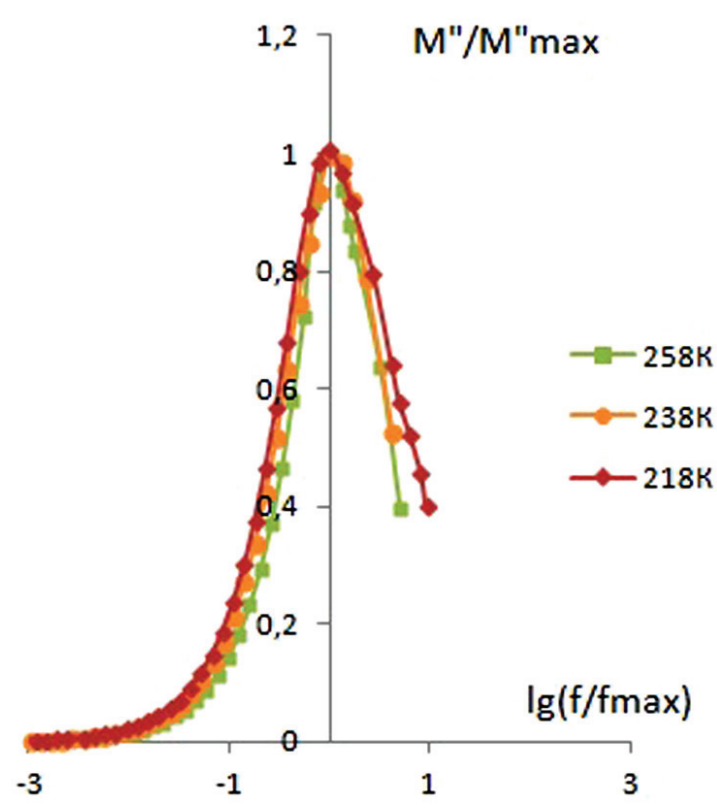

Рис. 6. Изменение $M^{\prime \prime} / M_{\max }^{\prime \prime}$ от $\lg \left(f / f_{\max }\right)$ при различных температурах для $\mathrm{H}_{2} \mathrm{Sb}_{1.52} \mathrm{~V}_{0.48} \mathrm{O}_{6} \cdot \mathrm{nH}_{2} \mathrm{O}$

[Fig. 6. Variation of $M^{\prime \prime} / M_{\max }^{\prime \prime}$ on $\lg \left(f / f_{\max }\right)$ at different temperatures for $\left.\mathrm{H}_{2} \mathrm{Sb}_{1.52} \mathrm{~V}_{0.48} \mathrm{O}_{6} \cdot \mathrm{nH}_{2} \mathrm{O}\right]$

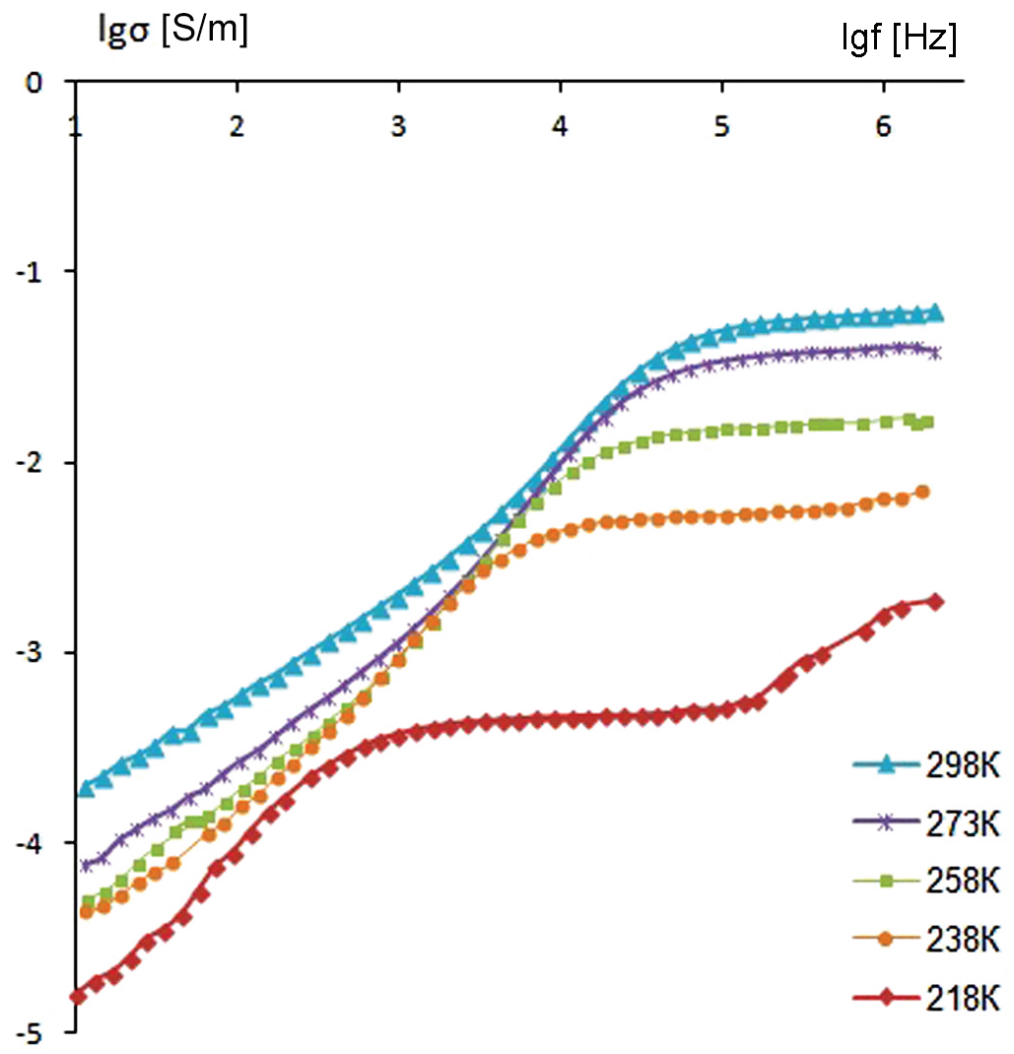

Рис. 7. Зависимость удельной проводимости от логарифма частоты при различных температурах для $\mathrm{H}_{2} \mathrm{Sb}_{1.52} \mathrm{~V}_{0.48} \mathrm{O}_{6} \cdot \mathrm{nH}_{2} \mathrm{O}$

[Fig. 7. Log-log plots of conductivity against frequency at different temperatures for $\mathrm{H}_{2} \mathrm{Sb}_{1.52} \mathrm{~V}_{0.48} \mathrm{O}_{6} \cdot \mathrm{nH}_{2} \mathrm{O}$ ] 


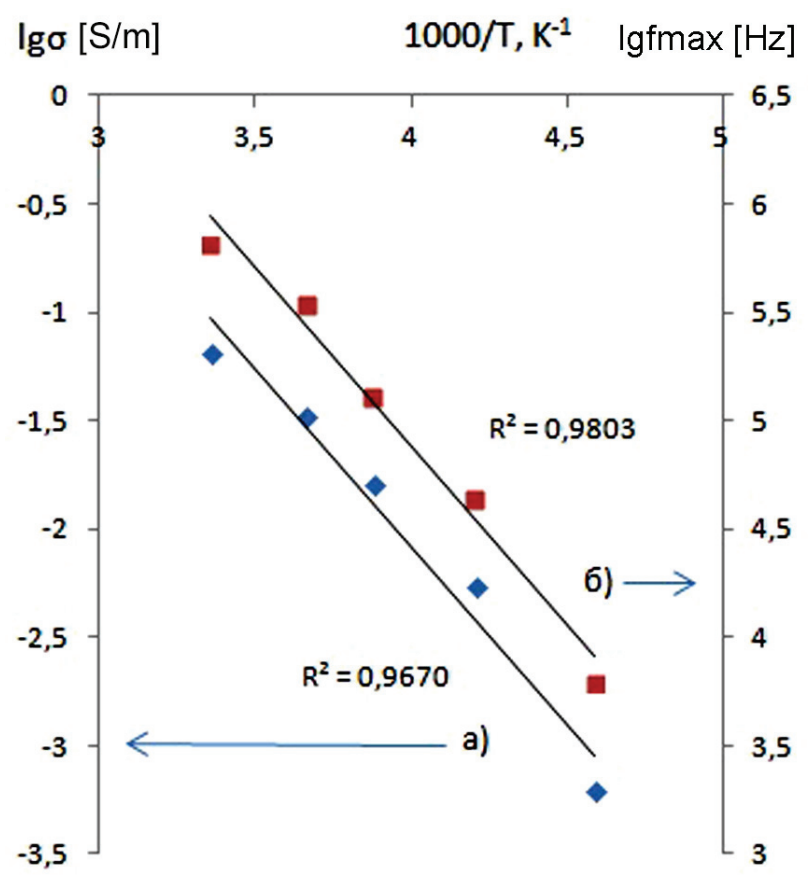

Рис. 8. Зависимость логарифма удельной проводимости (a) и логарифма частоты $\lg f_{\max }$ при котором фиксируется максимум на кривой $\operatorname{tg} \delta(b)$, от обратной температуры для

$$
\mathrm{H}_{2} \mathrm{Sb}_{1.52} \mathrm{~V}_{0.48} \mathrm{O}_{6} \cdot \mathrm{nH}_{2} \mathrm{O}
$$

[Fig. 8. Dependence of logarithm of the conductivity

(a) and of logarithm of the frequency $\lg f_{\max }$, at which the maximum is fixed on the curve $\operatorname{tg} \delta(b)$, at different temperatures for $\mathrm{H}_{2} \mathrm{Sb}_{1.52} \mathrm{~V}_{0.48} \mathrm{O}_{6} \cdot \mathrm{nH}_{2} \mathrm{O}$ ]

Основные результаты, полученные в настоящей работе, показывают, что введение ионов ванадия приводит к увеличению значений проводимости и уменьшению энергии активации. Наиболее заметные изменения фиксируются при введении максимально возможного количества ванадия. Так, для крайнего твердого раствора замещения $\mathrm{H}_{2} \mathrm{Sb}_{1.52} \mathrm{~V}_{0.48} \mathrm{O}_{6} \cdot \mathrm{nH}_{2} \mathrm{O}$ значение протонной проводимости, найденное по частотно независимому плато, при RH = 58 \% и 298 К составляет $66 \mathrm{mS} / \mathrm{m}$.

Наиболее распространенным механизмом транспорта заряда в конденсированных протонных системах является скоррелированный транспорт протонов вдоль определенной цепи «водородных связей». Поэтому увеличение значения протонной проводимости и уменьшение энергии активации допированных ионами ванадия образцов ПСК при пониженных температурах, могут быть связаны с изменением состава частиц. Известно, что элементарная ячейка ПСК состоит из отрицательно заряженных октаэдров $\left[\mathrm{SbO}_{3}\right]^{-}$, заряд которых компенсирует заряд протонов и ионов оксония [13]. При допировании ПСК ионами ванадия происходит замещение части сурьмы на ионы ванадия, при этом изменяются параметры структуры и степень взаимодействия протонов с отрицательно заряженным остовом [10]. Атом ванадия имеет меньшую электроотрицательность по сравнению с атомом сурьмы, что обуславливает уменьшение протонакцепторной способности аниона и энергии водородных связей [20]. При этом транспорт протонов осуществляется по системе водородных связей, образованных молекулами воды, расположенными в гексагональных каналах структуры типа пирохлора, и анионами кислорода октаэдра $\left[\mathrm{BO}_{3}\right]^{-}$, где $\mathrm{B}=\mathrm{V}, \mathrm{Sb}$, формирующего каркас структуры.

\section{выводы}

1.Исследована протонная проводимость ПСК при допировании ее ионами ванадия, показано, что замещение части ионов сурьмы каркаса структуры типа пирохлора ионами ванадия увеличивает протонную проводимость и уменьшает ее энергию активации.

2. Полученное значение протонной проводимости для крайнего твердого раствора $\mathrm{H}_{2} \mathrm{Sb}_{1.52} \mathrm{~V}_{0.48} \mathrm{O}_{6} \cdot \mathrm{nH}_{2} \mathrm{O}$ при $\mathrm{RH}=58 \%$ и 298 К составляет $66 \mathrm{mS} / \mathrm{m}$, а энергия активации проводимости $30 \pm 2 \mathrm{~kJ} / \mathrm{mol}$.

3. В допированных ионами ванадия ПСК проводимость осуществляется по системе водородных связей, образованных молекулами воды, расположенными в гексагональных каналах структуры типа пирохлора, и анионами кислорода октаэдра, формирующего каркас структуры. Введение ионов ванадия с меньшей электроотрицательностью приводит к уменьшению протоноакцепторной способности аниона и обеспечивает лучший транспорт протонов.

\section{ИСТОЧНИК ФИНАНСИРОВАНИЯ}

Работа выполнена при финансовой поддержке РФФИ проект № 18-33-00269.

\section{КОНФЛИКТ ИНТЕРЕСОВ}

Авторы декларируют отсутствие явных и потенциальных конфликтов интересов, связанных с публикацией настоящей статьи.

\section{СПИСОК ЛИТЕРАТУРЫ}

1. Стенина И. А., Ярославцев А. Б. Низко- и среднетемпературные протонпроводящие электролиты, «Неорганические материалы» // Неорганические материалы, 2017, т. 53(3), с. 241-251. DOI: $10.7868 / S 0002337 X 17030101$ 
2. Иванчев С. С., Мякин С. В. Полимерные мембраны для топливных элементов: получение, структура, модифицирование, свойства // Успехи химии, 2010, т. 79, с. 117-134.

3. Luo T., Abdu S., Wessling M. Selectivity of ion exchange membranes: A review // J. Membr. Sci., 2018, v. 555, p. 429. DOI: $10.1016 /$ j.memsci.2018.03.051

4. Фоменков А. И., Пинус И. Ю., Перегудов А. С., Зубавичус Я. В., Ярославцев А. Б., Хохлов А.Р. Протонная проводимость полиариленэфиркетонов с разной степенью сульфирования и ее повышение введением нанодисперсного кислого фосфата циркония // Высокомолекулярные соединения, Серия Б, 2007, т. 49(7), с. 1299-1305.

5. Prikhno I. A., Ivanova K. A., Don G. M., Yaroslavtsev A. B. Hybrid membranes based on short side chain perfluorinated sulfonic acid membranes (Inion) and heteropoly acid salts // Mendeleev Commun, 2018, v. 28(6), p. 657. DOI: 10.1016/ j.mencom.2018.11.033

6. Klestchov D., Burmistrov V., Sheinkman A., Pletnev R. Composition and structure of phases formed in the process of hydrated antimony pentoxide thermolysis // Journal of Solid State Chemistry, 1991, v. 94(2), p. 220. DOI: 10.1016/00224596(91)90186-L

7. Ярошенко Ф. А., Бурмистров В. А. Диэлектрическая релаксация и протонная проводимость полисурьмяной кристаллической кислоты при низких температурах // Электрохимия, 2015, т. 51(5), с. 455-461. DOI: 10.7868/ S0424857015050205

8. Ярошенко Ф. А., Бурмистров В. А. Исследование протонной проводимости полисурьмяной кислоты методом импедансной спектроскопии в интервале температур 370-480 K // Неорганические материалы, 2015, т. 51(8), с. 854-858. DOI: 10.7868/S0002337X15080205

9. Щелканова М. С., Пантюхина М. И., Калашнова А. В., Антонов Б. Д. Создание новых твердотельных электролитов на основе $\mathrm{Li}_{8-X} \mathrm{Zr}_{1-X} \mathrm{~V}_{X} \mathrm{O}_{6} / /$ Бутлеровские сообщения, 2014, т. 38(5), с. 96-102.

10. Коваленко Л. Ю., Бурмистров В. А., Лупицкая Ю. А., Ковалев И. Н., Галимов Д. М. Синтез твердых растворов $\mathrm{H}_{2} \mathrm{Sb}_{2-\mathrm{X}} \mathrm{V}_{\mathrm{x}} \mathrm{O}_{6} \cdot \mathrm{nH}_{2} \mathrm{O}$ со структурой типа пирохлора // Бутлеровские сообщения, 2018, т. 55(8), с. 24-30.

11. Коваленко Л. Ю., Бурмистров В. А., Лупицкая Ю. А. Влияние относительной влажности на протонную проводимость полисурьмяных кислот, допированных ионами ванадия // «Физикохимические процессы в конденсированных средах и на межфазных границах (ФАГРАН-2018)», мате- риалы VIII Всероссийской конференции с международным участием, 8-11 октября 2018, г. Воронеж, с. 524-525.

12. Malyshkina I. A., Makhaeva E. E., Gavrilova N. D., Khokhlov A. R. Peculiarities of low-frequency dielectric dispersion in polymer networks based on poly(methacrylic acid). Polymer Science. Series A, 2000, v. 42(8), pp. 325-328. URL: https:// elibrary.ru/item.asp?id=13345750

13. Клещев Д. Г. Механизм фазовых превращений при термолизе гидрата пентаоксида в интервале 470-730 K // Известия АН СССР. Heорганические материалы, 1987, т. 23(7), с. 11731176.

14. Armstrong R. D., Dickinson T., Willis P. M. The A. C. impedance of powdered and sintered solid ionic conductors // Electroanalytical Chem. Interfacial Electrochem, 1974, v. 53(3), pp. 389-405. DOI: 10.1016/S0022-0728(74)80077-X

15. Нифталиев С. И., Козадерова О. А., Ким К. Б., Матчина К. С. Изучение процесса переноса тока в системе гетерогенная ионообменная мембрана - раствор нитрата аммония // $\mathrm{Koн}^{-}$ денсированные среды и межфазные границы, 2016, т. 18(2), c. 232-240. URL: http://www.kcmf.vsu.ru/ resources/t_18_2_2016_007.pdf

16. Alvarez R., Guerrero F., Garcia-Belmonte G., Bisquert J. Study of the humidity effect in the electrical response of the $\mathrm{KSbMoO} 6$ ionic conductive ceramic at low temperature // Materials Sci. Eng., 2002, v. 90(3), pp. 291-295. DOI: 10.1016/s09215107(02)00004-1

17. Солодуха А. М., Либерман 3. А. Определение диэлектрических параметров керамики на основе дисперсии комплексного электрического модуля // Вестник ВГУ, Серия физика, математикa, 2003, № 2, c. 67-71. URL: http://www.vestnik. vsu.ru/pdf/physmath/2003/02/soloduha.pdf

18. Moti Ram, Chakrabarti S. Dielectric and modulus behavior of LiFe1/2Ni1/2VO4 ceramics // J. Phys. Chem. Solids, 2008, v. 69(4), pp. 905-912.DOI: 10.1016/j.jpcs.2007.10.008

19. Петьков В. И., Суханов М. В., Шипилов А. С., Куражковская В. С., Боровикова Е. Ю., Пинус И. Ю., Ярославцев А. Б. Синтез и свойства $\mathrm{LiZr}_{2}\left(\mathrm{AsO}_{4}\right)_{3}$ и $\mathrm{LiZr}_{2}\left(\mathrm{AsO}_{4}\right)_{x}\left(\mathrm{PO}_{4}\right)_{3-x} / /$ Неорганические материалы, 2014, т. 50(3), с. 285-294. DOI: 10.7868/S0002337X14030099

20. Краснов А. Г., Пийр И. В., Секушин Н. А., Бакланова Я. В., Денисова Т. А. Электрофизические свойства титанатов висмута со структурой типа пирохлора $\mathrm{Bi}_{1.6} \mathrm{M}_{x} \mathrm{Ti}_{2} \mathrm{O}_{7-\delta}(\mathrm{M}=\mathrm{In}, \mathrm{Li}) / /$ Электрохимия, 2017, т. 53(8), с. 972. DOI: 10.7868/ S0424857017080114 
UDC 544.6.018.47-039.6+546.865-31

DOI: https://doi.org/10.17308/kcmf.2019.21/758

Received 30.04.2019

Accepted 15.05.2019

\title{
DIELECTRIC RELAXATION AND PROTON CONDUCTIVITY OF POLYANTIMONIC ACID DOPED WITH VANADIUM IONS
}

\author{
(C) 2019 L. Yu. Kovalenko $\bowtie$, V. A. Burmistrov \\ Chelyabinsk State University \\ 129, Brat'ev Kashirinyh str., 454001 Chelyabinsk, Russian Federation
}

Abstract

Purpose. To improve the operating characteristics of proton-conducting polymer membranes, nanoparticles of heteropoly acids and hydrated oxides of polyvalent elements are being infused. Such membranes are used in condensers of huge capacity, memory devices, selective sensors, fuel cells and ion-selective membranes. One of the perspective heteropoly acids, crystallized within the pyrochlore-type structure (sp. gr. $\mathrm{Fd3m}$ ), is the polyantimonic acid $\mathrm{H}_{2} \mathrm{Sb}_{2} \mathrm{O}_{6} \cdot \mathrm{nH}_{2} \mathrm{O}$, $2<n<4$ (PAA). In a number of researches, it is shown that the proton-crystal cell interaction degree influences the proton-conducting properties of PAA. Therefore, PAA transport properties can be increased by means of partial substitution of antimony ions for ions with the approximate ionic radius, but different in the electronegativity values. It is expected that this kind of substitution within the pyrochlore-type structure may lead to the degradation of the interaction energy between protons with the anion sublattice and their migration within the system of hydrogen bonds, formed by water molecules. In this regard, the aim of this work is to synthesize and study the proton transport in PAA doped with vanadium ions.

Methods and methodology. Substitutional solid solutions have been obtained with the help of the co-precipitation method. The X-ray phase analysis and the elemental analysis have been conducted in order to clarify the phase composition. Earlier in the range of works it has been shown that polyantimonic acid doping with vanadium ions within the pyrochlore-type structure has a concentration limitation: substitutional solid solution $\mathrm{H}_{2} \mathrm{Sb}_{2-x} \mathrm{~V}_{x} \mathrm{O}_{6} \cdot \mathrm{nH}_{2} \mathrm{O}$ is formed when $0<\mathrm{x}<0,48$. That is why the samples, containing 0 (PAA); $10(x=0.2) ; 20(x=0.40) ; 24(x=0.48)$ at. \% of vanadium have been chosen as subjects of the present research. By means of impedance spectroscopy method the proton-conducting properties with the relative humidity of $58 \%$, which was obtained by storing the measurement cell with the sample, placed inside the flask, above $\mathrm{NaBr}$ saturated solution surface, have been studied. A specially designed cell was used that represented a flat capacitor with graphite electrodes, between which the studied powder was compacted. In order to study the conductivity under the lower temperatures the cell, previously held within 24 hours in the flask with $\mathrm{RH}=58 \%$, was placed into a thermostat, allowing to vary the temperature from 218 to $298 \mathrm{~K}$ by cooling with solid carbon dioxide. According to the measuring data of the actual variable $Z^{\prime}$ and the imaginary variable $Z^{\prime \prime}$ of the complex impedance parts the dielectric properties have been determined.

Results. Synthesized samples represented green-coloured powders with the coherent-scattering region sizes, according to the X-ray spectral analysis, of $40 \mathrm{~nm}$. On the X-ray diffraction patterns there is an identical set of diffraction maximums. However, with the increase of vanadium quantity there is a shift into a wide diffraction angles' area, which is proved by the reduction of unit cell parameters. Impedance samples hodograph for the temperature of $298 \mathrm{~K}$ and $\mathrm{RH}=58 \%$ represents a slightly stretched semicircumference and a straight line at a slight angle to the $\mathrm{X}$-axis. With the help of the equivalent circuit these dependencies are described with the model 'of grains and interlayers'.

Conclusions. Proton conductivity of polyantimonic acid doped with vanadium ions has been studied. It has been shown that the partial substitution of antimony ions within the pyrochloretype structure for ions with the lower electronegativity value increases proton conductivity and reduces its activation energy. In this case for the extreme solid substitution solution $\mathrm{H}_{2} \mathrm{Sb}_{1.52} \mathrm{~V}_{0.48} \mathrm{O}_{6} \cdot \mathrm{nH}_{2} \mathrm{O}$ the value of proton conductivity at $\mathrm{RH}=58 \%$ and $298 \mathrm{~K}$ is $66 \mathrm{mS} / \mathrm{m}$. According to the analysis of dielectric spectroscopy data at temperatures of $218-298 \mathrm{~K}$ the activation energy of conductivity is $30 \pm 2 \mathrm{~kJ} / \mathrm{mol}$, also, the proton transport mechanism is proposed. In doped PAA conductivity is carried out through the system of hydrogen bonds, formed by water

$\triangle$ Kovalenko Liliya Yu., e-mail: LKovalenko90@mail.ru 
molecules, located in hexagonal channels within the pyrochlore-type structure and oxygen anions of octahedron, forming the structural framework.

Keywords: solid electrolytes, pyrochlore-type structure, antimony compounds, polyantimonic acid, solid substitution solutions, proton conductivity.

\section{SOURCE OF FINANCING}

The study was supported by the Russian Foundation for Basic Research (grant No. 18-3300269)

\section{CONFLICT OF INTEREST}

The authors declare the absence of obvious and potential conflicts of interest related to the publication of this article.

\section{REFERENCES}

1. Stenina I. A., Yaroslavtsev A. B. Low- and intermediate-temperature proton-conducting electrolytes. Inorg. Mater. 2017.v. 53(3), pp. 253-262.DOI: 10.1134/ S0020168517030104

2. Ivanchev S. S., Myakin S. V. Polymer membranes for fuel cells: manufacture, structure, modification, properties. Russian Chemical Reviews, 2010, v. 79(2), pp. 101-117. DOI: 10.1070/RC2010v079n02ABE H004070

3. Luo T., Abdu S., Wessling M. Selectivity of ion exchange membranes: A review. J. Membr. Sci., 2018, v. 555, pp. 429-454. DOI: 10.1016/j.memsci.2018. 03.051

4. Fomenkov A. I., Pinus I. Yu., Peregudov A. S., Zubavichus Ya. V., Yaroslavtsev A. B., Khokhlov A. R. Proton conductivity of poly(arylene ether ketones) with different sulfonation degrees: Improvement via incorporation of nanodisperse zirconium acid phosphate. Polymer Science Series B, 2007, v. 49(7-8), pp. 177-181. DOI: 10.1134/S1560090407070032

5. Prikhno I. A., Ivanova K. A., Don G. M., Yaroslavtsev A.B. Hybrid membranes based on short side chain perfluorinated sulfonic acid membranes (Inion) and heteropoly acid salts. Mendeleev Commun, 2018,v. 28(6), pp. 657-658. DOI: 10.1016/j.mencom.2018. 11.033

6. Klestchov D., Burmistrov V., Sheinkman A., Pletnev R. Composition and structure of phases formed in the process of hydrated antimony pentoxide thermolysis. Journal of Solid State Chemistry, 1991, v. 94(2), pp. 220-226. DOI: 10.1016/0022-4596(91)90186-L

7. Yaroshenko F. A., Burmistrov V. A. Dielectric relaxation and protonic conductivity of polyantimonic crystalline acid at low temperatures. Russian Journal of Electrochemistry, 2015, v. 51(5), pp. 391-396. DOI: 10.1134/S1023193515050195

8. Yaroshenko F. A., Burmistrov V. A. Proton conductivity of polyantimonic acid studied by impedance spectroscopy in the temperature range 370-480 K. Inorg. Mater., 2015, v. 51(8), pp. 783-787. DOI: 10.1134/ S0020168515080208
9. Shchelkanova M. S., Pantyukhina M. I., Antonov B. D., Kalashnova A. V. Produce new solid electrolytes based on the $\mathrm{Li}_{8-x} \mathrm{Zr}_{1-x} \mathrm{~V}_{x} \mathrm{O}_{6}$ system. Butlerov Communications, 2014, v. 38(5), pp. 96-102. URL: https://butlerov.com/stat/reports/details. asp?lang=ru\&id=15798 (in Russ.)

10. Kovalenko L. Yu., Burmistrov V. A., Lupitskaya Yu. A., Kovalev I. N., Galimov D. M. Synthesis of the solid solutions $\mathrm{H}_{2} \mathrm{Sb}_{2-x} \mathrm{~V}_{x} \mathrm{O}_{6} \cdot \mathrm{nH}_{2} \mathrm{O}$ with the pyrochlore-type structure. Butlerov Communications, 2018, v. 55(8), pp. 24-30. URL: https://butlerov.com/stat/reports/details.asp?lang=ru\&id=30164 (in Russ.)

11. Kovalenko L. Yu., Burmistrov V. A., Lupitskaya Yu.A. Vliyanie otnositel'noy vlazhnosti na protonnuyu provodimost' polisur'myanykh kislot, dopirovannykh ionami vanadiya [Effect of relative humidity on the proton conductivity of poly-antimony acids doped with vanadium ions]. "Physico-chemical processes in condensed media and interphase boundaries" (FAGRAN2018)", materials of the 8th All-Russian Conference with international participation, October 8-11, 2018, Voronezh, pp. 524-525. URL: https://elibrary.ru/item. asp?id=36837531. (in Russ.)

12. Malyshkina I. A., Makhaeva E. E., Gavrilova N. D., Khokhlov A. R. Peculiarities of low-frequency dielectric dispersion in polymer networks based on poly(methacrylic acid). Polymer science. Series A, 2000, v. 42(8), pp. 325-328. URL: https://elibrary.ru/item. asp?id $=13345750$

13. Kleschev D. G. Mekhanizm fazovykh prevrashcheniy pri termolize gidrata pen-taoksida $\mathrm{v}$ intervale $470-730 \mathrm{~K}$ [The mechanism of phase transformations during thermolysis of pentoxide hydrate in the range of 470-730 K]. News of the Academy of Sciences of the USSR. Inorganic materials, 1987, v. 23(7), pp. 1173 -1176. (in Russ.)

14. Armstrong R. D., Dickinson T., Willis P. M. The A. C. impedance of powdered and sintered solid ionic conductors. Electroanalytical Chem. Interfacial Electrochem, 1974, v. 53(3), pp. 389. DOI: 10.1016/ S0022-0728(74)80077-X

15. Niftaliev S. I., Kozaderova O. A., Kim K. B., Matchin K. S. Research of current transfer process in the system heterogeneous ion-exchange membrane - ammonium nitrate solution. Condensed Matter and Interphases, 2016, v. 18(2), pp. 232-240. URL: http://www. kcmf.vsu.ru/resources/t_18_2_2016_007.pdf (in Russ.)

16. Alvarez R., Guerrero F., Garcia-Belmonte G., Bisquert J. Study of the humidity effect in the electrical response of the KSbMoO6 ionic conductive 
ceramic at low temperature // Materials Sci. Eng., 2002, v. 90(3), pp. 291-295. DOI: 10.1016/s09215107(02)00004-1

17. Solodukha A. M., Lieberman Z. A. Opredelenie dielektricheskikh parametrov keramiki na osnove dispersii kompleksnogo elektricheskogo modulya [Determination of dielectric parameters of ceramics based on the dispersion of a complex electrical module]. Vestnik VSU, Series of Physics, Mathematics, 2003, no. 2, pp. 67-71. URL: http://www.vestnik.vsu.ru/pdf/ physmath/2003/02/pitanov.pdf. (in Russ.)

18. Moti Ram, Chakrabarti S. Dielectric and modulus behavior of LiFe1/2Ni1/2VO4 ceramics. J. Phys.
Chem. Solids, 2008, v.69(4), pp.905-912.DOI:10.1016/ j.jpcs.2007.10.008

19. Pet'Kov V. I., Sukhanov M. V., Shipilov A. S., Kurazhkovskaya V. S., Borovikova E. Y., Pinus I. Y., Yaroslavtsev A. B. Synthesis and properties of $\operatorname{LiZr}_{2}\left(\mathrm{AsO}_{4}\right)_{3}$ and $\mathrm{LiZr}_{2}\left(\mathrm{AsO}_{4}\right) \times\left(\mathrm{PO}_{4}\right)_{3-x^{*}}$. Inorg. Mater., 2014, v. 50(3), pp. 263-272. DOI: 10.1134/S0020168514030091

20. Krasnov A. G., Piir I. V., Sekushin N. A., Baklanova Y. V., Denisova T. A. Electrophysical properties of bismuth titanates with the pyrochlore structure $\mathrm{Bi}_{1.6} \mathrm{M}_{x} \mathrm{Ti}_{2} \mathrm{O}_{7-\delta}(\mathrm{M}=\mathrm{In}, \mathrm{Li})$. Russian Journal of Electrochemistry, 2017, v. 53(8), pp. 866-872. DOI: 10.1134/ S1023193517080122
Коваленко Лилия Юрьевна - ассистент кафедры химии твердого тела и нанопроцессов, Челябинский государственный университет, Челябинск, Российская Федерация; e-mail: lkovalenko90@mail.ru. ORCID iD 0000-0002-9187-6934.

Бурмистров Владимир Александрович - д. фм. н., профессор, декан химического факультета, Челябинский государственный университет, Челябинск, Российская Федерация; e-mail: burmistrov@csu.ru. ORCID iD 0000-0002-7862-6017.
Kovalenko Liliya Yu. - assistant of the Department of Solid State Chemistry and Nanoprocesses, Chelyabinsk State University, Chelyabinsk, Russian Federation, e-mail:1kovalenko90@mail.ru. ORCID iD 0000-0002-9187-6934.

Burmistrov Vladimir A. - Dr. Sc. (Phys.Math.), Professor, Dean of Chemical Department, Chelyabinsk State University, Chelyabinsk, Russian Federation; e-mail: burmistrov@csu.ru. ORCID iD 0000-0002-7862-6017. 